\title{
Energy Scan Results at Belle
}

\author{
Junhao Yin for the Belle Collaboration ${ }^{* \dagger}$ \\ Institute of High Energy Physics, CAS, 19B Yuquan Road, Shijingshan District, Beijing, China \\ E-mail: yinjh@ihep.ac.cn
}

In this report, we present the most recent results on the measurement on the cross sections of $e^{+} e^{-} \rightarrow \pi^{+} \pi^{-} \Upsilon(n S),(n=1,2,3), e^{+} e^{-} \rightarrow \pi^{+} \pi^{-} h_{b}(1,2 P), e^{+} e^{-} \rightarrow B_{s}^{(*)} \bar{B}_{s}^{(*)}$, and $e^{+} e^{-} \rightarrow$ $\pi^{+} \pi^{-} \pi^{0} \chi_{b J}(J=0,1,2)$ based on energy scan data. Further analysis are also discussed.

XVII International Conference on Hadron Spectroscopy and Structure - Hadron2017

25-29 September, 2017

University of Salamanca, Salamanca, Spain

* Speaker.

$\dagger$ This work is supported in part by National Natural Science Foundation of China (NSFC) under contract Nos. 11475187 and 11521505; Key Research Program of Frontier Sciences, CAS, Grant No. QYZDJ-SSW-SLH011; and the CAS center for Excellence in Particle Physics (CCEPP). 


\section{Introduction}

As an effective theory, the potential model works well in describing the heavy quarkonia states. However, for the bottomnium beyond the $B_{s} B_{s}$ threshold, some problems occurred. The $\Upsilon(10860)$ state, which is historically called $\Upsilon(5 S)$, has unexpected higher mass than the prediction and higher rate of hadronic bottomonium transitions. The transition rate of $\Upsilon(5 S) \rightarrow \pi \pi \Upsilon(n S)$ is about two orders of magnitude larger than those in $\Upsilon(1,2,3 S)$ decays [1]. Meanwhile, the transition rate of $\Upsilon(5 S) \rightarrow \pi \pi h_{b}$ is at the same order of magnitude despite this process requiring a $b$-quark spinflip [2]. There are several hypothesis trying to explain these phenomena, for example, assuming the $\Upsilon(5 S)$ consist of 4 four quarks in the wave function[3]. Another puzzle is the finding that the peak of $R_{\Upsilon(n S) \pi^{+} \pi^{-}} \equiv \sigma\left(\Upsilon(n S) \pi^{+} \pi^{-}\right) / \sigma_{\mu^{+} \mu^{-}}^{0}$ near $\Upsilon(5 S)$ occurs at a mass $9 \pm 4 \mathrm{MeV} / \mathrm{c}^{2}$ higher than that derived from $R_{b} \equiv \sigma(b \bar{b}) / \sigma_{\mu^{+} \mu^{-}}^{0}[4]$.

With a massive data around $\Upsilon(5 S)$ collected at Belle detector based on KEKB collider, including 3 major points very near at $\Upsilon(5 S)$ peak, 22 points with integrated luminosity of $1 \mathrm{fb}^{-1}$ per point, and 61 points with integrated luminosity of $50 \mathrm{pb}^{-1}$ per point, Belle collaboration is able to measure the Born cross sections around $\Upsilon(5 S)$ and $\Upsilon(6 S)$ region with improved precision.

\section{2. $R_{\Upsilon(n S)}$ and $R_{b}^{\prime}$ measurements}

Belle report the measurement of the total cross section for $e^{+} e^{-} \rightarrow \pi^{+} \pi^{-} \Upsilon(n S),(n=1,2,3)$ and $b \bar{b}$ quark pairs is based on all the datasets [5]. The distribution of $R_{\Upsilon(n S) \pi^{+} \pi^{-}}$, calculated using $N_{\Upsilon(n S) \pi^{+} \pi^{-}} /\left(\mathscr{L}_{i} \mathscr{B}\left(\Upsilon(n S) \rightarrow \mu^{+} \mu^{-}\right) \sigma_{\mu^{+} \mu^{-}}^{0}\left(\sqrt{s_{i}}\right)\right)$, and the fit are shown in Fig. 1. A coherent sum of two $S$-wave Breit-Wigner amplitudes for $\Upsilon(5 S)$ and $\Upsilon(6 S)$ and a constant, plus an incoherent constant is used to fit the $R_{\Upsilon(n S) \pi^{+} \pi^{-}}$distribution. The $\Upsilon(5 S)$ and $\Upsilon(6 S)$ masses, width$\mathrm{s}$, and the relative phases are allowed to float. The fitting results are $M(\Upsilon(5 S))=(10891.1 \pm$ $\left.3.2_{-1.7}^{+0.6}\right) \mathrm{MeV} / \mathrm{c}^{2}, \Gamma(\Upsilon(5 S))=\left(53.7_{-5.6-5.4}^{+7.1+1.3}\right) \mathrm{MeV}, M(\Upsilon(6 S))=\left(10.987 .5_{-2.5-2.1}^{+6.4+9.0}\right) \mathrm{MeV} / \mathrm{c}^{2}$, and $\Gamma(\Upsilon(6 S))=\left(61_{-19-20}^{+9}\right) \mathrm{MeV}$.

The $R_{b}^{\prime}$ distribution, calculated using $R_{b}^{\prime} \equiv R_{b, i}-\sum \sigma_{I S R, i} / \sigma_{\mu^{+} \mu^{-}, i}^{0}$, is shown in the bottom plot in Fig. 1. Note that the measurements yield the visible cross sections and include neither corrections due to the ISR events containing $b \bar{b}$ final states above $B \bar{B}$ threshold nor the vacuum polarization necessary to obtain the Born cross section. The background from the $q \bar{q}$, where $q=u, d, s, c$, are subtracted based on the data taken at $10.52 \mathrm{GeV}$ with a scale of $1 / s$, where $\sigma_{b \bar{b}}=0$. With the same fitting method, the fit results are shown in Fig. 1. The fitting range is restricted to $10.82-11.05 \mathrm{GeV}$ to avoid complicated threshold effects below $10.8 \mathrm{GeV}$. A large resonance-continuum interference is reflected in this fit. Although the fitting results on resonance parameters are consistent with those from $R_{\Upsilon(n s) \pi^{+} \pi^{-}}$fitting, the validity of using a flat continuum in the $R_{b}^{\prime}$ fit is brought into questions by incompatibilities between the fitted amplitudes for $R_{b}^{\prime}$. and $R_{\Upsilon(n S) \pi^{+} \pi^{-}}$.

\section{Energy Scan of $e^{+} e^{-} \rightarrow \pi^{+} \pi^{-} h_{b}(1,2 P)$ and evidence for charged bottomonium-like State}

Belle also report the analysis on $e^{+} e^{-} \rightarrow \pi^{+} \pi^{-} h_{b}(1,2 P)$ using the full $\Upsilon(5 S)$ and $\Upsilon(6 S)$ scan data [6]. The measured Born cross section of $e^{+} e^{-} \rightarrow \pi^{+} \pi^{-} h_{b}(1,2 P)$ are shown in Fig. 2. A simultaneous fit with coherent sum of two Breit-Wigner amplitudes is performed to the cross sections 


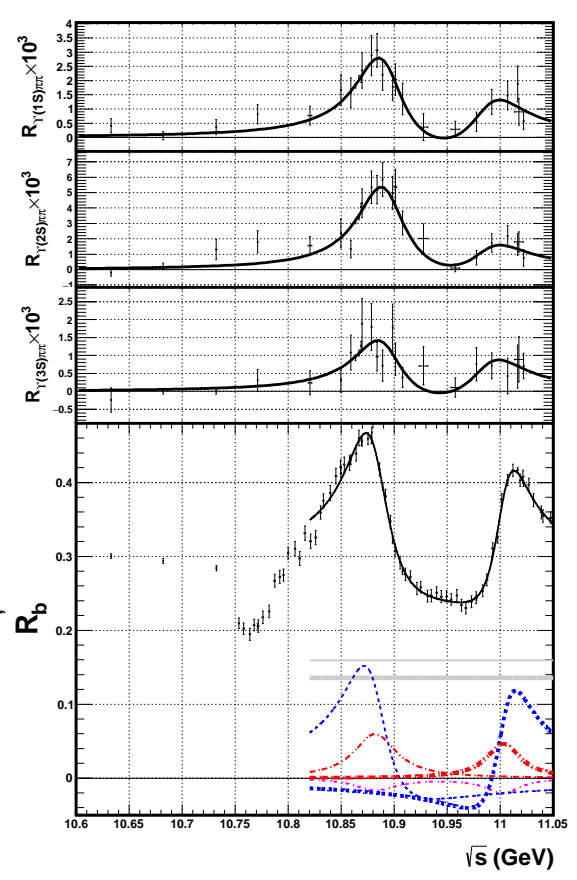

Figure 1: $R\left(\Upsilon(n S) \pi^{+} \pi^{-}\right)$data with fit results for $\Upsilon(1 S) ; \Upsilon(2 S) ; \Upsilon(3 S)$; and $R_{b}^{\prime}$ data with components of fit: $\Upsilon(5 S)$ (thin) and $\Upsilon(6 S)$ (thick).

and a continuum component is considered in the systematic uncertainty. The masses and widths of the measured resonances are $M=\left(10884.7_{-3.4-1.0}^{+3.6+8.9}\right) \mathrm{MeV} / \mathrm{c}^{2}$ and $\Gamma=\left(40.6_{-8.0-19.1}^{+12.7+1.1}\right) \mathrm{MeV}$ for $\Upsilon(5 S) ; M=\left(10999.0_{-7.8-1.0}^{+7.3+16.9}\right) \mathrm{MeV} / \mathrm{c}^{2}$ and $\Gamma=\left(27_{-11-12}^{+27+5}\right) \mathrm{MeV}$ for $\Upsilon(6 S)$. The significances of $h_{b}(1 P)$ and $h_{b}(2 P)$ signals in the combined five data points around the $\Upsilon(6 S)$ resonance are $3.5 \sigma$ and $5.3 \sigma$, respectively. The intermediate resonant substructures from $\Upsilon(6 S) \rightarrow \pi^{+} \pi^{-} h_{b}(1,2 P)$ are checked via yielding $h_{b}$ in bins of the recoiling mass of $\pi$. The distribution of the recoiling mass of $\pi$ are shown in Fig. 2(a)(b) as well as the fitting function. The fitting results imply that the processes of $\Upsilon(6 S) \rightarrow \pi^{+} \pi^{-} h_{b}(1,2 P)$ are proceed entirely via the intermediate isovector $Z_{b}(10610)$ and $Z_{b}(10650)$. The hypothesis that only $Z_{b}(10610)$ is produced is excluded at the level of 3.3 standard deviations, while the hypothesis that only $Z_{b}(10650)$ is produced is not excluded at a significant level.

\section{Study of $e^{+} e^{-} \rightarrow B_{s}^{(*)} \bar{B}_{s}^{(*)}$ Production}

Belle also report the production of $e^{+} e^{-} \rightarrow B_{s}^{(*)} \bar{B}_{s}^{(*)}$ processes based on $\Upsilon(5 S)$ on resonance data and the energy dependent cross section based on the scan data [7]. Clear signal of $e^{+} e^{-} \rightarrow$ $\Upsilon(5 S) \rightarrow B_{s}^{(*)} \bar{B}_{s}^{(*)}$ are observed, while no statistically significant signal of $e^{+} e^{-} \rightarrow \Upsilon(6 S) \rightarrow B_{s}^{(*)} \bar{B}_{s}^{(*)}$ processes. The relative production rate of $B_{s}^{*} \bar{B}_{s}^{*}, B_{s}^{*} \bar{B}_{s}^{(*)}$, and $B_{s} \bar{B}_{s}$ final states at $\sqrt{s}=10.866 \mathrm{GeV}$, which is very close to the $\Upsilon(5 S)$ peak, is measured to be $7: 0.856 \pm 0.106$ (stat.) \pm 0.053 (syst.) : $0.645 \pm$ 0.094 (stat. $)_{-0.033}^{+0.030}$ (syst.). An angular analysis of the $B_{S}^{*} \bar{B}_{s}^{*}$ final state produced at $\Upsilon(5 S)$ peak is also 

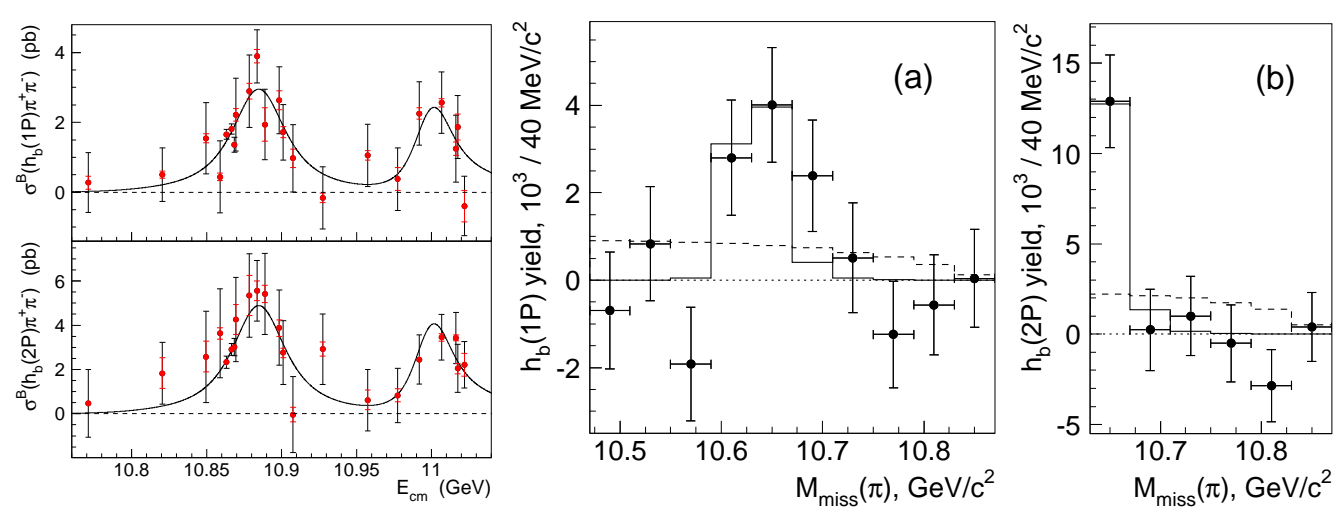

Figure 2: The cross sections for $e^{+} e^{-} \rightarrow h_{b}(1 P) \pi^{+} \pi^{-}$(left top) and $e^{+} e^{-} \rightarrow h_{b}(2 P) \pi^{+} \pi^{-}$(left bottom) as function of center-of-mass energy, and the solid curves are the fit results. The efficiency corrected yields of $h_{b}(1 p) \pi^{+} \pi^{-}$(a) and $h_{b}(2 P) \pi^{+} \pi^{-}$(b) as functions of $\pi$ recoiling mass for the combined data samples of five energy points in the $\Upsilon(6 S)$ region, where points represent data, solid lines represent the fit results, and the dashed lines represent the fit results with a phase space distribution.

performed. The fraction of $S=0$ component is determined to be $r \equiv \frac{a_{0}^{2}}{a_{2}^{2}+a_{0}^{2}}=0.175 \pm 0.057_{-0.018}^{+0.022}$. Energy dependent cross section of $e^{+} e^{-} \rightarrow B_{s}^{(*)} \bar{B}_{s}^{(*)}$ processes are shown in Fig. 3, which reveals a strong signal of $\Upsilon(5 S)$ resonance while no significant signal of $\Upsilon(6 S)$ resonance.
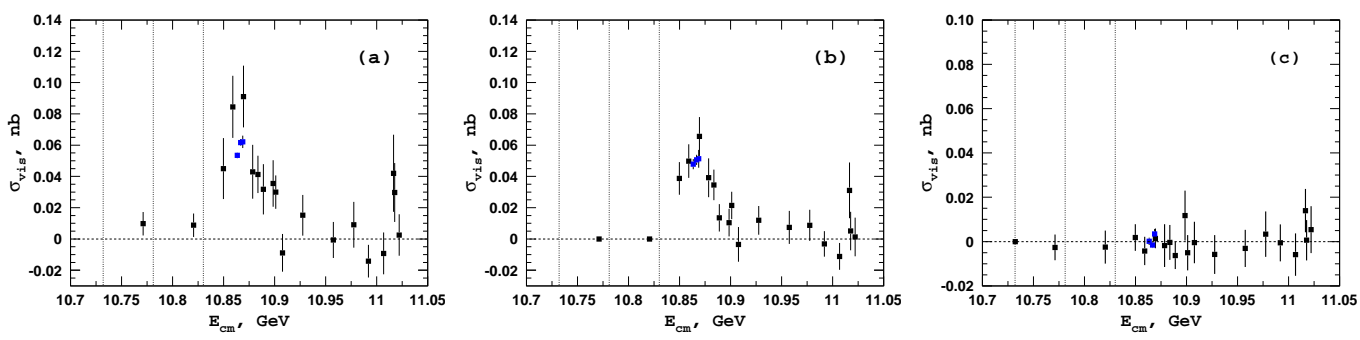

Figure 3: Cross section for the (a) total $e^{+} e^{-} \rightarrow B_{s}^{(*)} \bar{B}_{s}^{(*)}$; (b) $e^{+} e^{-} \rightarrow B_{s}^{*} \bar{B}_{s}^{*}$ only; (c) tagged $B$ meson momentum sideband region. Vertical lines show the $B_{s} \bar{B}_{s}, B_{s} \bar{B}_{s}^{*}$, and $B_{s}^{*} \bar{B}_{s}^{*}$ thresholds, respectively.

\section{Study of $e^{+} e^{-} \rightarrow \pi^{+} \pi^{-} \pi^{0} \chi_{b J}$}

The processes $e^{+} e^{-} \rightarrow \pi^{+} \pi^{-} \pi^{0} \chi_{b J}$ are studied at $\sqrt{s}=10.867 \mathrm{GeV}$ [8]. The $\gamma \Upsilon(1 S)$ invariant mass distribution in the whole $\pi^{+} \pi^{-} \pi^{0}$ mass region after events selection is shown in Fig. 4 (left). Clear peaking signal of $\chi_{b 1}$ and $\chi_{b 2}$ are observed while $\chi_{b 0}$ is not evident. An unbinned maximum likelihood fit is applied and the solid curve shows the fit results. The Born cross section of $e^{+} e^{-} \rightarrow$ $\pi^{+} \pi^{-} \pi^{0} \chi_{b J}$ are $<3.1 \mathrm{pb}^{-1},=(0.90 \pm 0.11 \pm 0.13) \mathrm{pb}^{-1}$, and $=(0.57 \pm 0.13 \pm 0.08) \mathrm{pb}^{-1}$ for $J=$ 0,1 , and 2 , respectively. Both $\omega \chi_{b J}$ events and non- $\omega \chi_{b J}$ events are observed in the $\pi^{+} \pi^{-} \pi^{0}$ mass spectrum. The mass spectra of $\gamma \Upsilon(1 S)$ within and outside $\omega$ signal region are also shown in Fig. 4 (middle, right). The measured Born cross section $\sigma\left(e^{+} e^{-} \rightarrow \omega \chi_{b J}\right)<1.9 \mathrm{pb}^{-1},=(0.76 \pm 0.11 \pm$ 
$0.11) \mathrm{pb}^{-1}$, and $=(0.19 \pm 0.11 \pm 0.08) \mathrm{pb}^{-1}$ for $J=0,1$, and 2, respectively; while $\sigma\left(e^{+} e^{-} \rightarrow\right.$ $\left.\pi^{+} \pi^{-} \pi_{\text {non }-\omega}^{0} \chi_{b J}\right)<2.3 \mathrm{pb}^{-1},=(0.25 \pm 0.07 \pm 0.06) \mathrm{pb}^{-1}$, and $=(0.30 \pm 0.11 \pm 0.14) \mathrm{pb}^{-1}$. This is the first observation of hadronic transitions between $\Upsilon(5 S)$ and $\chi_{b 1,2}$ bottomonium states. The measured ratio of the cross section of $e^{+} e^{-} \rightarrow \omega \chi_{b 2}$ to $\omega \chi_{b 1}$ is $0.38 \pm 0.16$ (stat.) \pm 0.09 (syst.), which is significantly lower than the expectation of 1.57 from the heavy quark symmetry.
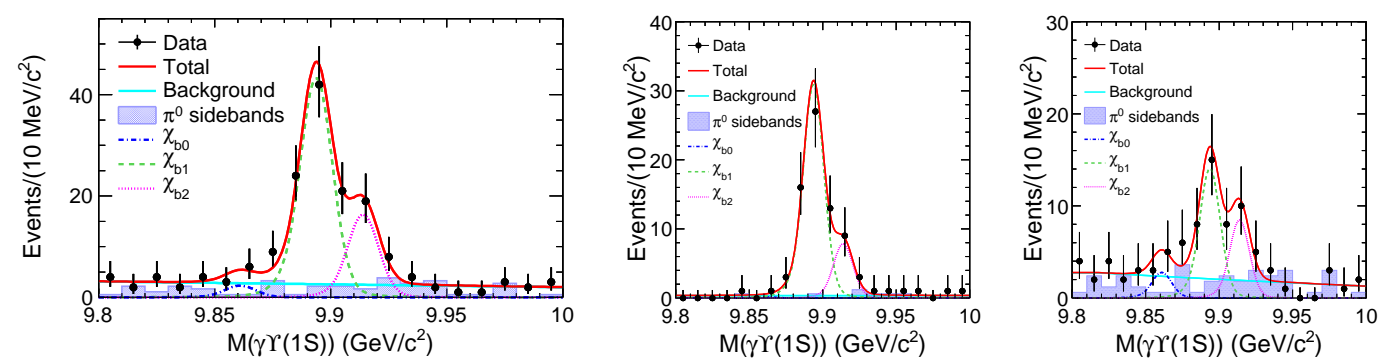

Figure 4: The $\gamma \Upsilon(1 S)$ invariant mass distributions for selected $e^{+} e^{-} \rightarrow \pi^{+} \pi^{-} \pi^{0} \gamma \Upsilon(1 S)$ candidate events in (left) whole $\pi^{+} \pi^{-} \pi^{0}$ mass regoin, (middle) the $\omega$ signal region, and (right) outside of $\omega$ signal region. The solid curves are the best fit for the total fit the background shape; the dash-dotted, dashed and dotted curves represent the $\chi_{b 0}, \chi_{b 1}$, and $\chi_{b 2}$ signals, respectively.

\section{Summary}

The cross section measurements of $e^{+} e^{-} \rightarrow \Upsilon(n S) \pi^{+} \pi^{-}, b \bar{b}, \pi^{+} \pi^{-} h_{b}(n P)$, and $B_{s}^{(*)} \bar{B}_{s}^{(*)}$ between 10.63 and $11.02 \mathrm{GeV}$ are performed and the resonance parameters of $\Upsilon(5 S)$ and $\Upsilon(6 S)$ mesons are obtained. It is interesting that the resonance peak of $\Upsilon(5 S)$ from $B_{s}^{(*)} \bar{B}_{s}^{(*)}$ mode is different from those of $\Upsilon(n S) \pi^{+} \pi^{-}$and $h_{b}(n P) \pi^{+} \pi^{-}$modes, this is still a puzzle to us. The processes $\Upsilon(5 S) \rightarrow \pi^{+} \pi^{-} \pi^{0} \chi_{b 1,2}$ are observed for the first time.

\section{References}

[1] K. F. Chen et al. [Belle Collaboration], Phys. Rev. Lett. 100, 112001 (2008).

[2] I. Adachi et al. [Belle Collaboration], Phys. Rev. Lett. 108, 032001 (2012).

[3] A. Ali, C. Hambrock and M. J. Aslam, Phys. Rev. Lett. 104, 162001 (2010) Erratum: [Phys. Rev. Lett. 107, 049903 (2011)]/PhysRevLett.104.162001, 10.1103/PhysRevLett.107.049903.

[4] K.-F. Chen et al. [Belle Collaboration], Phys. Rev. D 82, 091106 (2010).

[5] D. Santel et al. [Belle Collaboration], Phys. Rev. D 93, no. 1, 011101 (2016).

[6] A. Abdesselam et al. [Belle Collaboration], Phys. Rev. Lett. 117, no. 14, 142001 (2016).

[7] A. Abdesselam et al., arXiv:1609.08749 [hep-ex].

[8] X. H. He et al. [Belle Collaboration], Phys. Rev. Lett. 113, no. 14, 142001 (2014). 